\title{
NON-LINEAR DIAMAGNETIC TRANSPORT \\ OF THE LARGE-SCALE MAGNETIC FIELD \\ IN THE SOLAR CONVECTION ZONE
}

\author{
V.N. KRIVODUBSKIJ \\ Kiev University Astronomical Observatory, Kiev 254053, Ukraine
}

\begin{abstract}
The mean magnetic field transport due to inhomogeneity of the turbulence intensity is considered taking the field back reaction on motion into account. In spite of the magnetic quenching, the downward diamagnetic pumping is still powerful enough to keep the fields of 3 to $4 \mathrm{kG}$ strength near the SCZ base against the magnetic buoyancy.
\end{abstract}

Key words: Sun: magnetic fields, diamagnetism, escape problem

The turbulent fluid with inhomogeneous effective diffusivity $\eta_{T} \simeq \frac{1}{3} v l$ behaves as a diamagnet and in the kinematic regime in the mixing-length approach (the characteristic scale of velocity fluctuation is equal to the mixing-length scale $l$ ) transports the mean magnetic field with the effective velocity $\mathbf{V}_{\mu}=-\nabla \eta_{T} / 2$ (Ruzmaikin and Vainshtein, 1978) ( $v$ is the rms turbulent velocity). In nonlinear regime the diamagnetic transport velocity, $V_{D}$, depends on magnetic induction $B$ through the parameter $\beta=c_{a} / v=B / B_{e q}$,

$$
\begin{gathered}
V_{D}(\beta)=6 V_{\mu} \Psi_{D}(\beta) \\
\Psi_{D}(\beta)=\frac{1}{\beta^{2}}\left(\frac{\arctan \beta}{\beta}-\frac{1}{1+\beta^{2}}\right) .
\end{gathered}
$$

Here $c_{a}=B / \sqrt{4 \pi \rho}$ is the local Alfvén velocity, $B_{e q}=\sqrt{4 \pi \rho v^{2}}$ is the equipartition magnetic induction, $\rho$ is the material density, $\Psi_{D}(\beta)$ is the quanching function in the mixing-length approximation. The non-linear diamagnetic transport velocity, $V_{D}(\beta)$, decreases steadily with increasing magnetic field strength and becomes almost negligible in strong field case $(\beta \gg 1)$ (Kichatinov and Rüdiger, 1992). This rises the quastion of whether the (nonlinear) diamagnetism can indeed serve to store magnetic flux near the SCZ bottom against the buoyant escape.

The velocities of diamagnetic transport of the large-scale magnetic field in the kinematic approach, $V_{\mu}$, for the SCZ models of Baker and Temesvary (1966) and Spruit (1977) were calculated earlier (Krivodubskij, 1984; Schüssler, 1984). In lower half of the SCZ the diamagnetism acts against magnetic buoyancy. Here we consider the non-linear turbulent diamagnetic transport for the SCZ model by Stix (1989). In the SCZ, $\beta \ll 1$ or $\beta<1$, i.e., the magnetic fields are relatively weak. The small $\beta$ asymptotic of the above quanching function is

$$
\Psi_{D}(\beta) \simeq\left(\frac{1}{6}-\frac{\beta^{2}}{5}\right) .
$$

The values of the steady-state mean magnetic field, $B \simeq \beta B_{e q}$, kept in deep layers of the SCZ against the buoyant rise with velocity $V_{B}(\beta) \simeq \frac{l}{H_{p}} \frac{v}{\gamma} \frac{\beta^{2}}{15}\left(H_{p}\right.$ is the 
pressure scale height, $\left.\gamma=\frac{5}{3}\right)$, the parameter $\beta \simeq\left[5 V_{\mu} /\left(\frac{l}{I I_{p}} \cdot \frac{v}{3 \gamma}+6 V_{\mu}\right)\right]^{1 / 2}$, and the quanching function, $\Psi_{D}(\beta)$, were estimated from the equilibrium condition,

$$
\mathbf{V}_{B}(\beta)+\mathbf{V}_{D}(\beta)=0
$$

The estimated values which we obtained for the near-bottom region of the SCZ are: $\beta \simeq 0.5-0.8, \Psi_{D}(\beta) \simeq 0.01-0.10, B \simeq 3000-4000 G$. The velocity of non-linear turbulent diamagnetic pumping of magnetic fields to the SCZ bottom is about $V_{D} \simeq 3 \times 10^{2} \mathrm{~cm} / \mathrm{s}$ (in kinematic approach $V_{\mu} \simeq 4 \times 10^{3} \mathrm{~cm} / \mathrm{s}$ ).

Thus the non-linear regime of the turbulent diamagnetic pumping, as well as the kinematic one, provides suppression of the buoyant losses of magnetic flux and may store magnetic fields of about 3000 - $4000 \mathrm{G}$ near the SCZ bottom which site is supposed favourable for the solar dynamo.

\section{References}

Baker, N., Temesvary, S. 1966, Tables of Convective Stellar Envelope Models, 2nd cd. N.Y.: NASA. Kichatinov, L.L., Rüdiger, G.: 1992, 'Magnetic-field advection in inhomogenous turbulence', $A s$ tron. Astropnys., 260, 494.

Krivodubskij, V.N.: 1984, Astron. Zh., 61 , 354 (Sov. Astronomy, 28 , 205).

Ruzmaikin, A.A., Vainshtein, S.I.: 1978, Astrophys. Space Sci., 57, 195.

Schüssler, M. 1984, The Hydromagnetics of the Sun, ESA SP-220, ESA Scientific \& Technical Publications Branch ESTEC: Noordwijk, p.67.

Spruit, H.C.: 1977, Thesis, Univ. of Utrecht, p.17.

Stix, M. 1989, The Sun, Springer-Verlag: Berlin, Heidelberg, New York, London, Paris, Tokyo, p.200. 